\title{
Polyneuropathy associated with IgM monoclonal gammapathy with anti-MAG
}

INSERM

\section{Source}

INSERM. (1999). Orphanet: an online rare disease and orphan drug data base.

Polyneuropathy associated with IgM monoclonal gammapathy with anti-MAG.

ORPHA:639

Polyneuropathy associated with IgM monoclonal gammapathy (MG) with anti-MAG

(myelin-associated-glycoprotein) activity is a demyelinating polyneuropathy characterized clinically by sensory ataxia, tremor, paresthesia, and impaired gait. 